\title{
The Application of Advanced Nanostructured Film in Electrosurgical Device: Anti-Sticking Behavior and Thermal Injury
}

Keng-Liang Ou ${ }^{1,2,3,4}$ and Han-Yi Cheng ${ }^{1,2,3 *}$

${ }^{1}$ Graduate Institute of Biomedical Materials and Tissue Engineering, Taipei Medical University, Taipei 110, Taiwan

${ }^{2}$ Research Center for Biomedical Devices and Prototyping Production, Taipei Medical University, Taipei 110, Taiwan

${ }^{3}$ Research Center for Biomedical Implants and Microsurgery Devices, Taipei Medical University, Taipei 110, Taiwan

${ }^{4}$ Department of Dentistry, Taipei Medical University-Shuang Ho Hospital, New Taipei City 235, Taiwan

\begin{abstract}
Background: Minimally invasive surgery is performed using an endoscope and other instruments including the electrosurgical units. However, concerns including surgical smoke, tissue sticking, and thermal injury are remaining in electro surgery.
\end{abstract}

Aims: Accordingly, a newly developed electrosurgical electrode coating with hydrogenated Cu-incorporated diamond-like carbon (DLC-Cu) film is purposed to improve the instrument performance.

Methods: The morphologies of DLC-Cu surfaces were characterized using transmission electron microscopy, scanning electron microscopy and atomic force microscopy. In this study, lesions were made on the liver lobes of adult rats, using a monopolar electrosurgical unit equipped with untreated stainless steel electrodes or treated-electrodes. Animals were sacrificed for evaluations at $0,3,7$, and 28 days post-operatively.

Results: Treated-electrodes generate less sticking tissues and adhesive blood cells. Thermography revealed the surgical temperature in liver tissue from the treated -electrode was significantly lower than the untreated-electrode. Total injury area of livers treated with treated-electrodes was significantly smaller than the untreated-electrodes treatment. Moreover, treated-electrodes caused a relatively smaller area of lateral thermal injury, a smaller area of fibrotic tissue, and a faster process of remodeling than the untreated-electrodes. Western blot analysis showed that rats treated with treated-electrode expressed lower levels of NF-KB, caspase-3, and MMP-9 than untreated-electrode. Immunofluorescence staining for caspase-3 revealed the untreated-electrode caused more serious injury.

Conclusions: This study reveals that the plating of electrodes with hydrogenated Cu-incorporated diamond-like carbon film is an efficient method for improving the performance of electrosurgical units. However, more tests must be carried out to confirm these promising findings in human patients.

Keywords: Minimally invasive surgery; Monopolar electrosurgery; Thermal injury; Biomedical coating; Tissue sticking; In vivo test

\section{Introduction}

Many traditional open surgical approaches are replaced by minimally invasive surgery (MIS) in a number of disciplines including general surgery, surgical oncology, colorectal surgery, pediatric surgery, thoracic surgery, upper and lower gastrointestinal tract surgery, obesity surgery, hepatobiliary surgery, and in other solid organs [1]. Compared with the open surgery, a MIS has the advantages such as reduced pain, less tissue injury, quicker return of oral intake, shorter hospitalization, and improved cosmetic appearance [2]. Some studies focused on the stress response to MIS, and an improved immune response was found for the procedures [3]. Moreover, MIS also increases operating room efficiency [4]. Hence MIS is growing remarkably over the past two decades, and the growth is continuously.

MIS is generally performed in company with an endoscope or laparoscope, and other instruments are inserted into the patient body through small incisions. An endoscope with multitasking platform was also developed [5]. Electrosurgery is one of the widely used endoscopic tools in MIS [6]. Monopolar or bipolar electrosurgical unit equipped with various types of electrodes can achieve desired surgical effects such as incision, ablation, cauterization, or coagulation. However, several concerns including tissue sticking, thermal injury, and surgical smoke are remaining in electrosurgery [7]. During an electrosurgical procedure, the heated tissues may adhere to the hot electrode. This increases the electrical resistance and hinders energy delivery to the targeted tissue, as well as making the process less accurate and more time-consuming. Adherent tissue that is not removed will eventually become carbonized charred tissue, and the surgeon may have to periodically suspend the operation to clean the electrode. In addition, when the electrode is removed for cleaning, the sticking tissue may tear adjacent tissue, resulting in unnecessary bleeding. The lateral spread of thermal energy from electrosurgical procedures can also affect surrounding tissues, leading to inadvertent damage to vital structures, delayed wound healing, and increased postoperative pain. Surgical smoke is another problem caused by heated electrodes $[8,9]$. Surgical smoke is generated when tissue is heated and cellular fluid is vaporized by the delivering energy of the electrosurgery, which may reduce the visibility of internal operating field [10]. The smoke has also been shown to contain noxious chemicals which are hazard to patients $[11,12]$. Other pitfalls including direct coupling, insulation failure, and

${ }^{*}$ Corresponding author: Han-Yi Cheng, Graduate Institute of Biomedical Materials and Tissue Engineering, Taipei Medical University, Taipei 110, Taiwan, Tel: 886-2 27361661/5401; Fax: 886-2-27395524; E-mail: chytmu@gmail.com

Received March 30, 2015; Accepted April 25, 2015; Published May 15, 2015

Citation: Ou KL, Cheng HY (2015) The Application of Advanced Nanostructured Film in Electrosurgical Device: Anti-Sticking Behavior and Thermal Injury. J Nanomed Nanotechnol 6: 291. doi:10.4172/2157-7439.1000291

Copyright: (c) 2015 Ou KL, et al. This is an open-access article distributed under the terms of the Creative Commons Attribution License, which permits unrestricted use, distribution, and reproduction in any medium, provided the original author and source are credited. 
capacitive coupling are reported for using monopolar electrosurgery during MIS [13].

In order to improve the performance of electrosurgical unit, many commercial electrodes are treated with surface modification to improve the thermal conductivity and anti-adhesive property. The plating materials include gold or silver (Conmed, Utica, NY), ceramic (ERBE, Marietta, GA), silicide (Valletlab, Tyco, Boulder, Co), Teflon/ Cr (Megadyne, Draper, UT), fluoride (Valletlab, Tyco, Boulder, Co) etc. Compared with the prototype electrode (stainless steel), surgeons with these modified electrodes have an improvement in anti tissueadhesion, clearance and durability. Therefore, the surface modification for the electrode can improve the instrument performance efficiently.

Diamond-like carbon (DLC) film is a substance with well smoothness, high hardness, low frictional coefficient, resistance to oxidation and corrosion, high thermal conductivity, and mechanical stability in biological environments [14]. DLC can be easily doped and alloyed with different elements because of its amorphous structure. In addition, DLC has well biocompatibility [15]. These properties make DLC applied in biomedical felids widely in recent years [16]. Singh et al. reported the anti-adhesive property for DLC against glial cell and fibroblast [17]. Zhang et al. also showed a remarkable decrease of platelet adhered on the DLC film compared to the Chrono flex or glass [18]. In particular, our latest research [19] demonstrated that the embedded nano- $\mathrm{Cu}$ particles did not change the microstructure transformation behavior of the hydrogenated $\mathrm{Cu}$-incorporated diamond-like carbon (DLC-Cu) films. Moreover, DLC-Cu films were nontoxic to osteoblast cells (MG-63) and fibroblast cells (NIH3T3) and were biocompatible. Nano-Cu particles have an important role in the antibacterial mechanisms of DLC-Cu films. Metallic $\mathrm{Cu}$ elements (probably $\mathrm{Cu}^{+}$ions or nano- $\mathrm{Cu}$ particles) can diffuse from the film and efficiently kill bacteria by destroying their cell walls and membranes thereby causing their cytoplasm to leak. a-C:H films with a Cu content of $27.8 \pm 3.4$ at $\%$ and above have superior antibacterial properties against both Gram-positive bacterium Staphylococcus aureus ATCC6538P and the Gram-negative bacterium Escherichia coli ATCC8739. Therefore, the DLC-Cu film can be considered a promising antibacterial coating for use in biomedical applications and for minimally invasive surgery devices. In this study, DLC-Cu film was proposed to deposit on the surface of electrode using a thin film coating technology. The electrode coated with the DLC-Cu thin film (DLC-Cu-SS) and untreated standard stainless steel (SS) electrodes induced lateral thermal injury characteristics were investigated clearly in rat liver by monopolar electrosurgery.

\section{Materials and Methods}

\section{Properties evaluation of the DLC-Cu-SS surface}

The atomic force microscopy transmission electron microscopy (TEM; JEM-2100), scanning electron microscopy (SEM; JEOL JSM6500F) and (AFM; Nanosurf-Mobil S) were employed to analyze the surface morphologies, roughness of the DLC-Cu-SS surfaces. Hardness of the DLC surface was measured by nano-indentation with a diamond Berkovich indenter (Asmec-UNAT-M); the average of the values obtained in ten tests was used in order to get good statistical averages. Moreover, wettability examinations were performed using the sessile drop method using a GBX DGD-DI contact angle goniometer. The liquid deionized water was adopted in the test. Contact angle measurements were measured using at least five drops for each sample in order to get good statistical averages.

\section{Procedure of DLC-Cu coating}

$\mathrm{DLC}-\mathrm{Cu}$ film was deposited in a radio frequency magnetron sputtering system. The procedure of deposition has been described elsewhere $[19,20]$. The commercially available SUS 304 stainless steel electrodes (blade types) were used as substrates. The substrates were degreased in an acetone bath at $80^{\circ} \mathrm{C}$, rinsed ultrasonically in ethanol, rinsed again in a deionized water bath, and air dried before being loaded into the chamber. After evacuating the chamber to a pressure of $2.0 \times$ $10^{-6}$ torr, the argon gas was introduced. The substrates were biased and argon ion bombarded for 5 minutes to remove surface contaminants. Subsequently, the $\mathrm{CH}_{4} / \mathrm{Ar}$ mixture gas was fed into the chamber. The $\mathrm{CH}_{4}$ was used as the carbon source. The glow discharge plasma was generated by applying a radio-frequency input power of $225 \mathrm{~W}$ on a $\mathrm{Cu}$ (99.999\% purity) sputtering target to dope $\mathrm{Cu}$ and synthesize the DLC-Cu films simultaneously. The Cu target was fixed at a distance of $60 \mathrm{~mm}$ directly above the substrate.

\section{Whole blood adhesion test}

Fresh whole blood was obtained from six healthy, non-smoker volunteers (mean age 25, range 23-29 y/o) with informed consent who had not taken any regimen 2 weeks prior to donation. Blood samples obtained from each donor were used to evaluate the blood adhesion test separately.

Unmodified stainless steel blade and DLC-Cu-blade were incubated with heparinized whole blood $(0.5 \mathrm{U} / \mathrm{ml})$ at $37^{\circ} \mathrm{C}$ for $5 \mathrm{~min}$ [21]. The electrodes were then washed gently using phosphate buffered saline (PBS). The adhered blood cells on blades were analyzed by using image analysis software (SPOT basic software, SPOT imaging solutions, MI, USA). Other blades were fixed in a $2.5 \%$ glutaraldehyde solution in PBS, followed by dehydration in a graded ethanol series, critical point drying (Sousimis, PVT-3B critical point dryer), sputter-coating with gold ion by an ion sputter (Joel, JFC-1100E Ion sputtering device, $1100 \mathrm{E}$ ), and examined using a scanning electron microscope.

\section{Animals}

The protocols for animal experiments were reviewed and approved by the Institutional Animal Care and Use Committee for Taipei Medical University (LAC-98-0009). Twenty-seven Sprague-Dawley rats (200-300 g, BioLASCO, Taiwan) were maintained in accordance with the guidelines for the care and use of laboratory animals. The animals were fed ad libitum and housed in an environment controlled for temperature, humidity, and light. Rats were marked to permit individual identification and were kept in their cages for 14 days prior to experimentation to allow for acclimatization to the laboratory conditions.

\section{Surgical procedure}

Conventional SS-blade electrodes and DLC-Cu-SS blade electrodes were used with an electrosurgical unit (ICC300H, EBRE, USA) in the animal study. The operation was performed under general anesthesia of isoflurane inhalation. Operations were performed under general anesthesia with inhaled isoflurane. After adequate skin preparation and sterilization, the liver was exposed through midline laparotomy with a retractor to avoid the liver injury. A DLC-Cu-SS blade or a SS blade was inserted to the liver lobe (3 $\mathrm{mm}$ in depth) to create an anterior lesion, using a fixed power setting $(40 \mathrm{~W})$ with a fixed activation times (4 seconds). The power setting and activation time were commonly used parameters and based on settings from a previous study [22]. Other 3 rats received laparotomy were served as a sham group. 
Citation: Ou KL, Cheng HY (2015) The Application of Advanced Nanostructured Film in Electrosurgical Device: Anti-Sticking Behavior and Thermal Injury. J Nanomed Nanotechnol 6: 291. doi:10.4172/2157-7439.1000291

Page 3 of 8

\section{Thermography}

The effects of the SS and DLC-Cu-SS electrodes on temperatures across the live lobe surface were determined from thermographs obtained with a thermal-imaging infrared camera (Advanced Thermo TVS-500EX, NEC Avio Technologies, Tokyo, Japan). The highest temperature was recorded for every test. After the thermography was recorded; the surgical wound was closed in layers. No antiinflammatory regimen was treated after surgery.

\section{Weight of sticking tissue}

The weights of each SS blade and DLC-Cu-SS blade were recorded perioperatively, and the weight of sticking tissue was calculated as the weight of blade post-operation minus the weight of blade preoperation.

\section{Postoperative pain assessment}

General physical conditions of rats were observed. Daily dietary intakes and weekly body weight changes were recorded to determine the postoperative pain indirectly.

\section{Injury area and histologic examination}

Animals were sacrificed on days 3, 7, 14, and 28 post-operation. The livers were removed and fixed in $10 \%$ formalin of neutral buffer. The samples were then dehydrated in a graded series of ethanol and embedded in paraffin wax. Consecutive sections were cut from the paraffin blocks into $5 \mu \mathrm{m}$ slide and deparaffinized. The sections were stained with hematoxylin and eosin (H\&E, 3008-1 and 3204-2, Muto, Japan) and Masson's trichrome staining to analyze the general morphology and fibrosis using a microscope (BX51, Olympus, Japan). The total injury areas caused by the SS or DLC-Cu-SS blades were measured using image analysis software. In addition, different histologic features were observed in the injury site.

\section{Western blot analysis}

The tissues surrounding the injury site of retrieved liver were harvested and examined separately for every rat. Samples were homogenized and lysed in an ice-cold protein lysis buffer and centrifuged at $13000 \mathrm{rpm}$ for 15 minutes at $4^{\circ} \mathrm{C}$. Protein concentrations of the lysates were determined using a Bio-Rad protein assay (Bio-Rad Laboratories, Hercules, CA, USA). The lysates $(20 \mu \mathrm{g} / \mathrm{lane})$ were resolved by sodium dodecyl sulfate (SDS)-polyacrylamide gel electrophoresis (PAGE) in 10\% gels and electroblotted onto a nitrocellulose membrane (Hybond $^{\mathrm{TM}}-\mathrm{C}$ Extra, Amersham Biosciences Corp., Hong Kong, China). The membrane was blocked for 30 minutes at room temperature in 3\% nonfat milk in Tris-buffered saline with $0.05 \%$ Tween 20 , and then incubated overnight at $4^{\circ} \mathrm{C}$ with the indicated primary antibodies against NF- $\kappa$ B (1:500, MAB3026, Chemicon, Millipore, Temecula, CA), or caspase-3 (1:200, AB1899, Millipore, Temecula, CA), or MMP-9 (1:500, Santa Cruz, CA) and then incubated with horseradish peroxidase-conjugated secondary antibodies for 2 hours at room temperature. After washing, the membrane was developed using chemiluminescent substrates (WBLUC0100, Millipore, Temecula, CA). Densiometric analysis of the gels was performed using Gel-Pro analyzer software, and the results were expressed relative to the density of $\beta$-actin (1:500, MAB1501, Chemicon, Millipore, Temecula, CA).

\section{Immunofluorescence staining}

For immunofluorescence staining, liver tissues were embedded in Cryomatrix (Thermo Shandon, Pittsburgh, PA, USA) and stored at $-80^{\circ} \mathrm{C}$. Cryostat sections (5-7 $\mathrm{mm}$ thickness) were fixed in a mixture of methanol and acetone $(1: 1)$ at $-20^{\circ} \mathrm{C}$ for 5 minutes and incubated in $1 \%$ Triton X-100 for another 5 minutes. Nonspecific activity was blocked by incubating the sections with $10 \%$ horse serum in a buffer containing $0.02 \%$ Tween 20 in PBS for 30 minutes. Sections were then incubated overnight with primary anti-Caspase- 3 at $4^{\circ} \mathrm{C}$. Antigen localization was indicated by a Cy5 dye (red fluorescence, 111-176-003, Jackson ImmunoResearch, West Grove, PA). Finally, samples were stained with 4',6-diamidino-2-phenyl-indole (DAPI, sc-3598, Santa Cruz Biotechnology, CA) for 7 minutes and examined using a fluorescent microscope. Normal liver tissues obtained from sham group were undergone identical procedures. Negative controls were processed identically, except that the primary antibodies were replaced with IgG.

\section{Statistical analysis}

Data were expressed as mean \pm standard error of the mean (SEM). All statistical analyses were performed using SPSS version 12.0 (SPSS, Inc, Chicago, IL). Values of $p<0.05$ were considered significant.

\section{Results}

\section{Characteristics of the DLC-Cu-SS treated surface}

Figure $1 \mathrm{~A}$ and $1 \mathrm{~B}$ shows a cross section of the DLC-Cu-SS thin film and the thickness was found to be approximately $250 \mathrm{~nm}$. From the TEM observation, it was evident that a homogenous and smooth surface with tiny nano $\mathrm{Cu}$ particles was deposited onto the surface of the substrate as depicted in Figure 1C; the average diameter of these nano $\mathrm{Cu}$ particles was found to be approximately $20-50 \mathrm{~nm}$. As $\mathrm{Cu}$ was doped, sphere-like nanoparticles with twins became embedded in the film as shown in Figure 1D. Figure $1 \mathrm{E}$ and $1 \mathrm{~F}$ showed the $2 \mathrm{D}$ and $3 \mathrm{D}$ surface morphologies of the DLC-Cu-SS surface using an AFM, which were acquired in a scanned range of $5 \times 5 \mu \mathrm{m}^{2}$. The average surface hardness values were significantly difference between
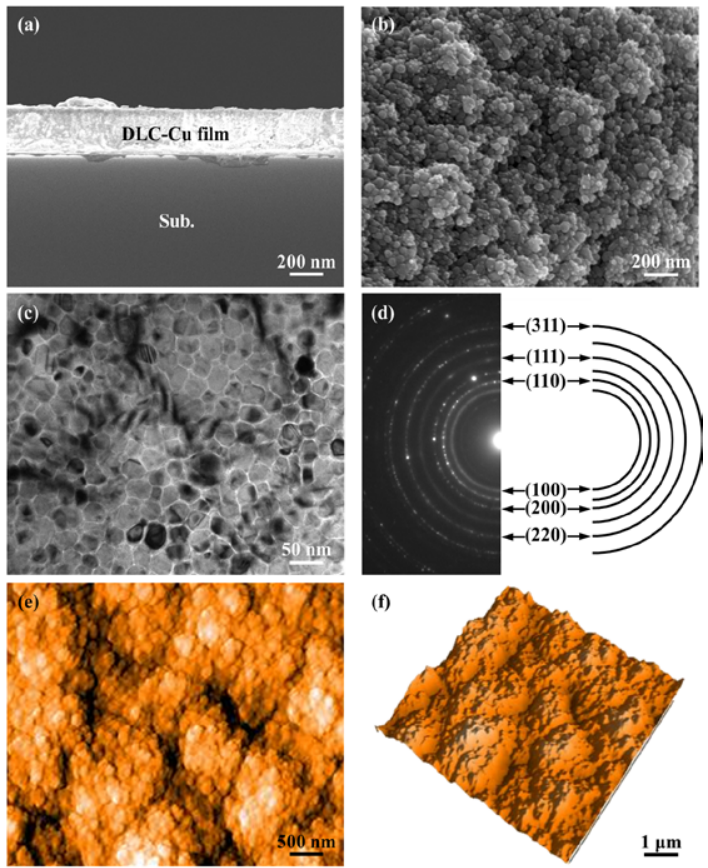

Figure 1: SEM (a) cross-section and (b) top view images of the DLC-Cu thin film (c) TEM and (d) the selected area electron diffraction pattern; AFM (e) 2D and (f) 3D images of the DLC-Cu thin film. 
Citation: Ou KL, Cheng HY (2015) The Application of Advanced Nanostructured Film in Electrosurgical Device: Anti-Sticking Behavior and Thermal Injury. J Nanomed Nanotechnol 6: 291. doi:10.4172/2157-7439.1000291

groups $(\mathrm{p}<0.05)$ as shown in Figure 2A. The contact angle of the DLC$\mathrm{Cu}$-SS treated surface was presented in Figure 2B. The SS surfaces showed the lowest value $(75.47 \pm 2.55)$, whereas DLC-Cu-SS surfaces exhibited comparative hydrophobicity, with a value of $97.25 \pm 1.87$. ANOVA revealed a significant difference between the SS and DLC$\mathrm{Cu}-\mathrm{SS}(\mathrm{p}<0.05)$. Clearly, the DLC-Cu-SS treated surface exhibited low roughness $(\sim 2.57 \mathrm{~nm})$ features to than that of untreated surface as shown in Figure 2C.

\section{DLC-Cu electrode and whole blood adhesion test}

The commercial SS and DLC-Cu-SS electrosurgical devices are shown in Figure 3. Blood cells adhered on blades were analyzed, and results show SS (33562 \pm 5347 pix, $n=5)$ had significantly higher amount of blood cells than that of DLC-Cu-SS $(23040 \pm 2448$ pix, $n=5$, $p<0.01)$. SEM further reveals the surface of SS blade had more adhesive red blood cells and fibrins than on the DLC-Cu-SS device.

\section{Electrosurgical procedure and thermography}

Figure 4 represents the electrosurgical procedure and the thermograph of SS and DLC-Cu-SS blades applied in liver lobes. The

(a)

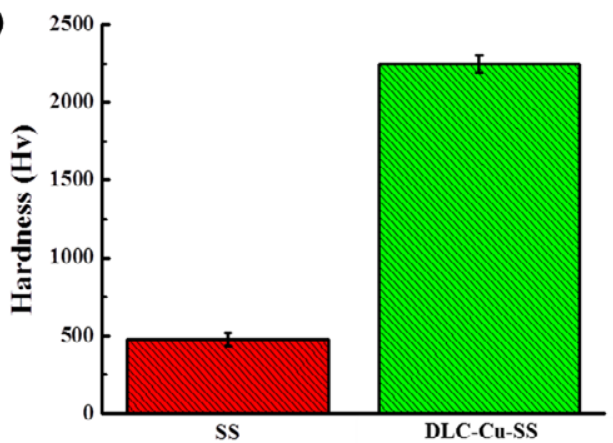

(b)

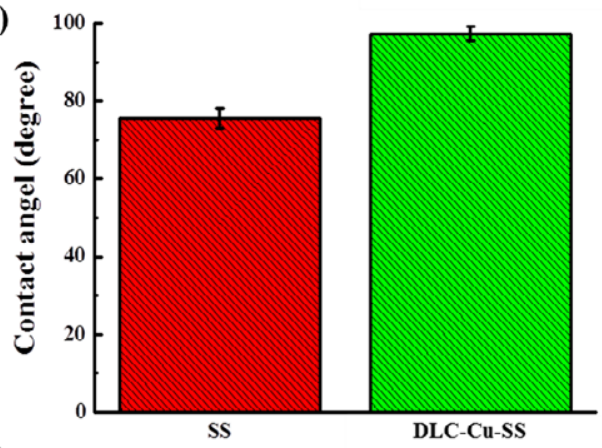

(c)

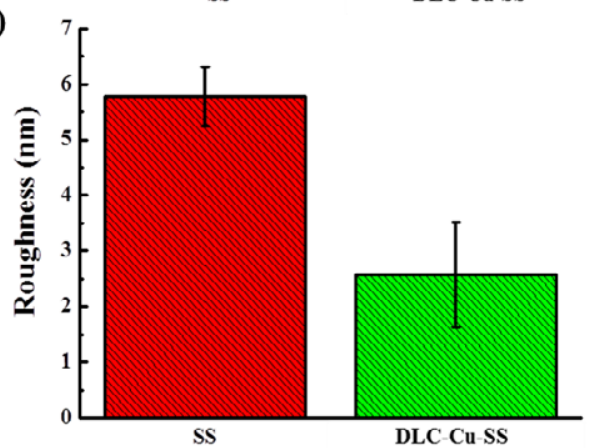

Figure 2: Statistics analysis of (a) hardness, (b) contact angel and (c) roughness in of the DLC-Cu thin film.

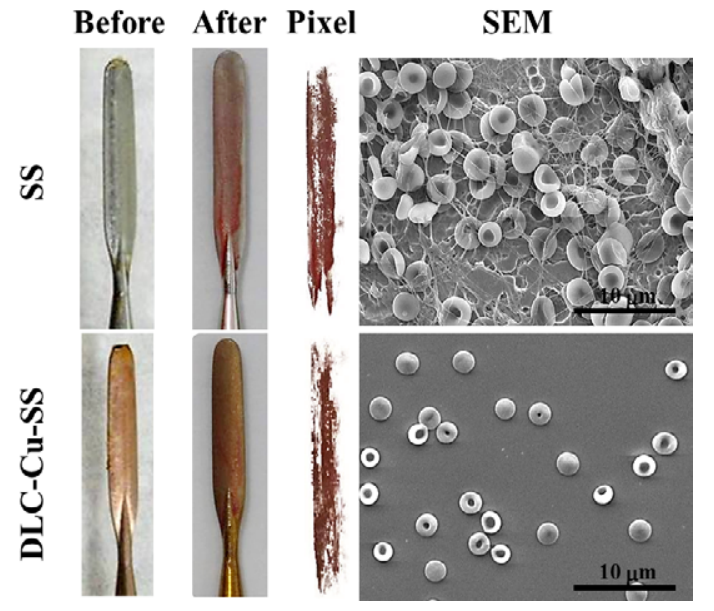

Figure 3: DLC-Cu-SS plated blade for whole blood adhesion tests and SEM reveals the surface of SS-blade had more adhesive red blood cells and fibrins than on the DLC-Cu-blade.

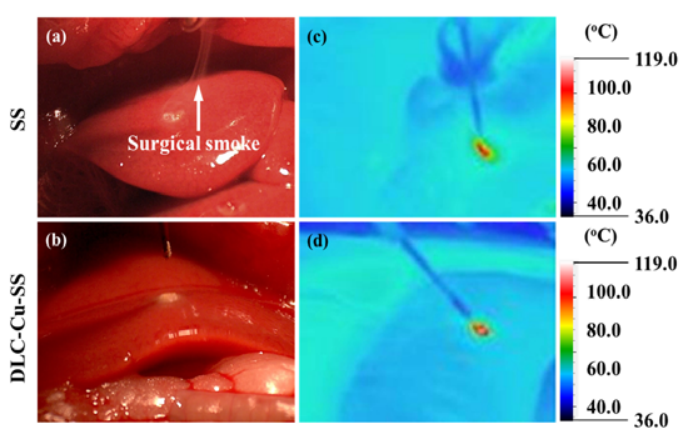

Figure 4: (a) Surgical smoke was noticed when using a SS-blade was used (b) No surgical smoke generated in DLC-Cu-SS group. (c) The highest recorded temperature was $161.83 \pm 18.47^{\circ} \mathrm{C}$ for the SS-blade group and (d) $134.12 \pm 9.41^{\circ} \mathrm{C}$ for DLC-Cu-blade group.

surgical smoke was noticed when using a SS blade, and the smoke still existed even when the electrode was removed (Figure 4A). However, the surgical smoke was not generated during the entire procedure for DLC-Cu-SS blade group (Figure 4B). The highest temperature recorded while applying an SS-blade was $161.83 \pm 18.47^{\circ} \mathrm{C}$ (Figure $4 \mathrm{C}$ ). The highest temperature recorded during DLC-Cu-SS blade application was $134.12 \pm 9.41^{\circ} \mathrm{C}$ (Figure 4D), which was significantly lower than that in the SS-blade group.

\section{Weight of sticking tissue}

The surface of the used SS blade (Figure 5A) shows a higher amount of adhesive tissue than that of DLC-Cu-SS blade (Figure 5B). When observing in stereomicroscope, the surface of SS-blade (Figure 5C) shows more charring tissues than that of DLC-Cu-SS blade (Figure 5D). The average weight of tissue sticking on the SS blade (4.37 \pm 0.52 $\mathrm{mg})$ was significantly higher than the DLC-Cu-SS blade $(1.02 \pm 0.21$ $\mathrm{mg}, \mathrm{n}=8, p<0.01$ ) (Figure 5E).

\section{Postoperative pain assessment}

The body weight changes reveal that all rats gained weight gradually (Figure 6). However, the body weight did not increased obviously during day 0 to day 10 for all groups. There was no significant 


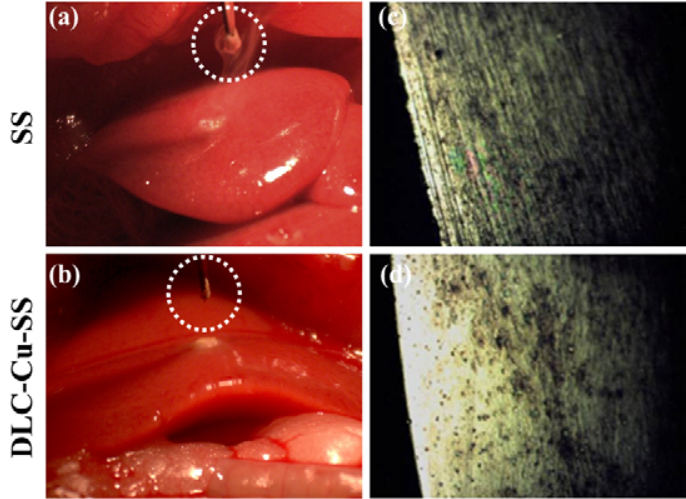

(e)

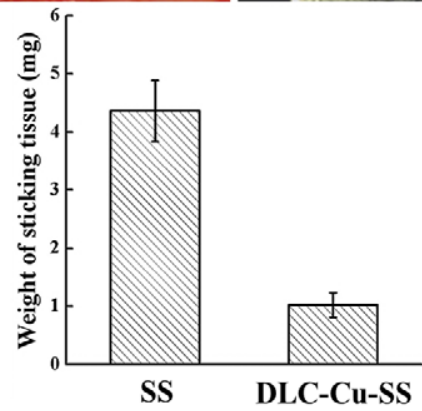

Figure 5: (a) Used SS electrode shows a higher amount of adhesive tissue than that of (b) DLC-Cu-SS electrode. (c) Surface of SS electrode shows more charring tissues than that of (d) DLC-Cu-SS electrode when observed using a stereomicroscope. (e) The weight of tissue sticking on the SS electrode was significantly higher than that on the DLC-Cu-SS electrode.

difference in body weight between groups. Daily dietary intakes also show the appetite of rats was decreased post-operatively (data not shown), and the oral intake of rats became normal after day 7 . These results reveal that rats received sham operation, SS electrode, or DLC$\mathrm{Cu}$-SS electrode treatments had same postoperative pain scores.

\section{Injury area and thermal injury assessment}

There was a significant difference in the total injury area caused by the SS and DLC-Cu-SS blades. The injury site in SS-blade group was significantly larger than that of DLC-Cu-SS blade at day 3 (Figure 7A). Although the total injury area for the SS group was decreased at day 7, the area was still significantly larger than the DLC-Cu-SS group. At days 14 and 28, the injury sites for both groups were decreased, but the area of injury for SS group remained significantly larger than the DLC-Cu-SS group. The total injury site was summarized in Figure 7B.

Masson's trichrome staining was used to examine the formation of fibrotic tissue. Minor fibrotic tissue was noticed in SS blade group in the boundary between thermal injury area and normal liver at day 3 (Figure 8A). No fibrotic tissue was evident for the DLC-Cu-SS blade group. At day 7, the fibrotic tissues were clearly found, and the fibrotic area (purple-blue color) of SS blade group was larger than that of DLC$\mathrm{Cu}$-SS blade group. Liver neogenesis was observed and the fibrotic area decreased at day 14 in DLC-Cu-SS blade group. However, SS blade group still shows large apoptosis area. The nodular foci of hepatocytes were formed in the apoptotic area for both groups at day 28, and the SS group still had a larger fibrotic area relative to the DLC-Cu-SS group.

The predominant injury area that resulted from the use of SS blades was different from the DLC-Cu-SS blades. Both types of blade caused tissue sticking and bleeding, direct injury, and thermal injury at day 3 . The area of blade adhesion and bleeding (red color) in SS group was significantly larger $(p<0.05)$ than that of DLC-Cu-SS group, and the SS blade also induced larger $(p<0.05)$ thermal injury areas (yellow color) than DLC-Cu-SS group at day 3 (Figure $8 \mathrm{~B}$ ). Some regions within the direct injury area (blue color) became apoptosis (orange color) in the SS group; and the characteristic of apoptosis was also observed in DLC$\mathrm{Cu}$-SS group at day 7 (Figure $8 \mathrm{C}$ ). The thermal injury areas (yellow color) were replaced by fibrotic tissues (purple color), and the fibrotic areas in SS group were larger than DLC-Cu-SS group $(p<0.01)$. On day 14, most of the direct injury areas (blue color) became apoptosis; the apoptotic tissues were predominant in SS group and the size was larger $(p<0.01$, Figure 8D) than DLC-Cu-SS group. The areas of direct injury and apoptosis were replaced by neogenesis area (pink color) in DLC-Cu-SS group. However, neogenesis area was rarely found in SS group ay day 14. Most apoptotic areas were replaced by regenerative hepatocyte foci in DLC-Cu-SS group at day 28 . However, rats treated with SS blades still showed a large area of apoptotic tissues (Figure 8E).

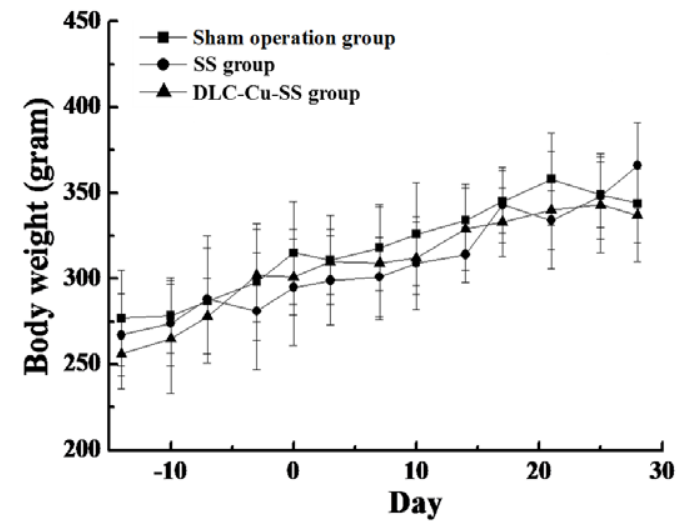

Figure 6: The body weight changes reveal that all rats gained weight gradually. There was no significant difference in body weight between groups. The result revealed that rats received sham operation, SS-electrode, or DLC-Cu-SS electrode treatments had same postoperative pain scores.

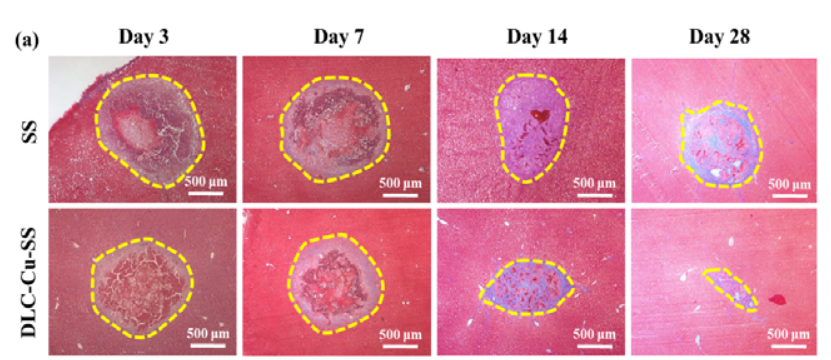

(b)

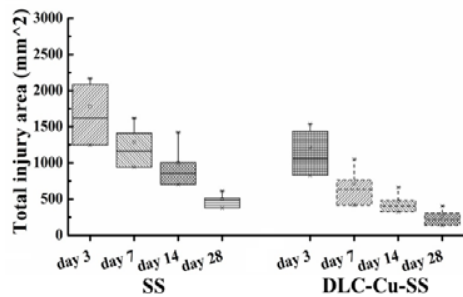

Figure 7: (a) The total injury area in SS electrode group was significantly larger than that for the DLC-Cu-SS blade group at day 3 , day 7 , day 14 , and day 28. The total injury site was summarized in (b). 
Citation: Ou KL, Cheng HY (2015) The Application of Advanced Nanostructured Film in Electrosurgical Device: Anti-Sticking Behavior and Thermal Injury. J Nanomed Nanotechnol 6: 291. doi:10.4172/2157-7439.1000291

A)

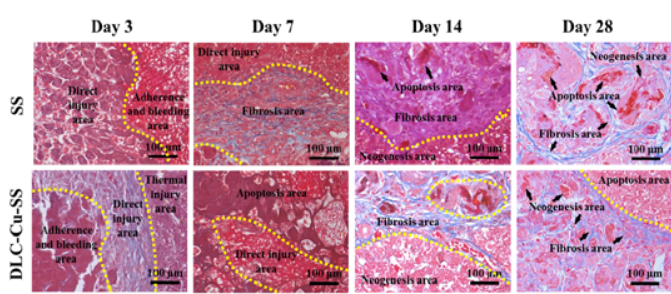

B)

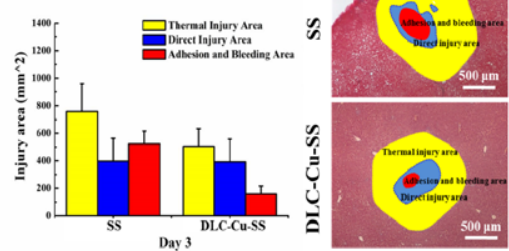

C)
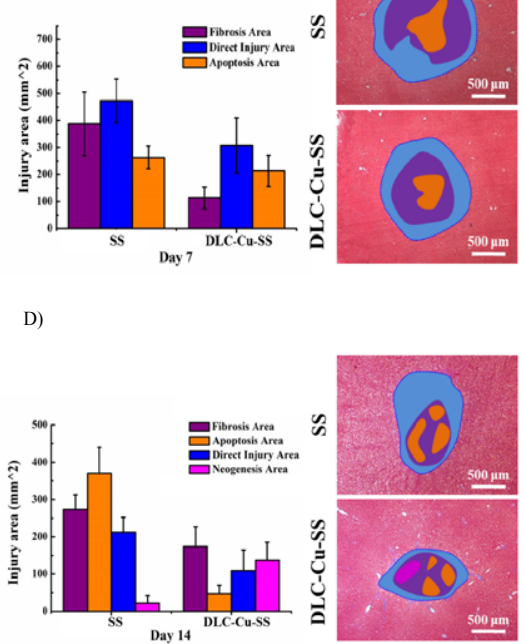

E)

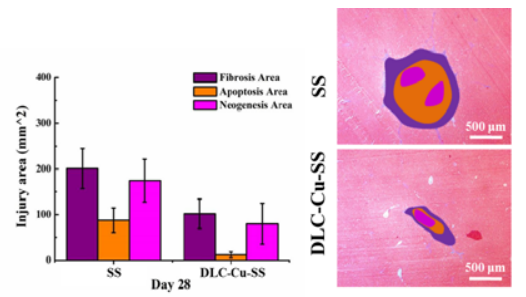

Figure 8: (a) Masson's trichrome staining showed minor fibrotic tissue in the boundary between thermal injury area and normal liver in the SS electrode group. No fibrotic tissue was evident for the DLC-Cu-SS blade group at day 3. At day 7 , the fibrotic tissues were clearly found in the SS electrode group, and the fibrotic area was larger than that of the DLC-Cu-SS electrode group. Liver neogenesis was minor with large area of apoptosis in the SS electrode group at day 14. DLC-Cu-SS electrode group had large area of neogenesis. Nodular foci of hepatocytes were formed in the apoptotic area for both groups at day 28 , and the SS electrode group still had a larger fibrotic area relative to the DLC-Cu-SS electrode group. Classification of the injury site by type at (b) day $3,(\mathrm{~b})$ day $7,(\mathrm{~d})$ day 14 , and (e) day 28.

\section{Western blot analysis}

Figure 9A represents the results of western blot and shows that the NF- $\kappa$ B was highly expressed in SS group at day 7 and day 14, and highly expressed in DLC-Cu-SS group at day 7. Densitomeric analysis of the gels revealed the expression of NF- $\mathrm{KB}$ protein in SS group was higher than that in DLC-Cu-SS group at day 3 and day 14 (Figure 9B). Caspase-3 was highly expressed at day 7 in both groups (Figure 9C). MMP-9 was highly expressed in SS blade relative to the DLC-Cu-SS group at day 14 (Figure 9D).

\section{Immunofluorescence staining}

Figure 10A shows tissues from rats treated with SS or DLC-Cu-SS blade, and normal live tissue sections. In the SS group, most caspase-3 positive cells were identified surrounding the injury site at day 3 (Figure 10B) and day 7 (Figure 10C). The caspase-3 positive cells were also identified surrounding the injury site at day 3 (Figure 10B) and day 7 (Figure 10D) for DLC-Cu-SS group. There were also few caspase-3 positive cells identified in normal rat liver (Figure 10E).

\section{Discussion}

Despite the widespread use of electrosurgical devices in MIS, the issues of surgical smoke, thermal injury and tissue sticking remain as disadvantages. Compared to the complex irrigation or automatic thermal control, coating a DLC-Cu film to modify the surface property of electrosurgical electrode is a relative simple and economical approach to improve the instrument performance. There is no need to modify the design of radio frequency (RF) generator or electrodes, but to deposit a DLC-Cu film on the electrodes using the thin film coating technology. Therefore, the surface modification for electrodes could be used in all types of electrosurgical devices for MIS.

To evaluate the anti-adhesive property for the DLC-Cu plating electrodes, whole blood test was used. Results obtained from SEM and image analysis reveal the DLC-Cu-SS electrode had less adhesion of blood cells relative to the SS-electrode. Moreover, the modified

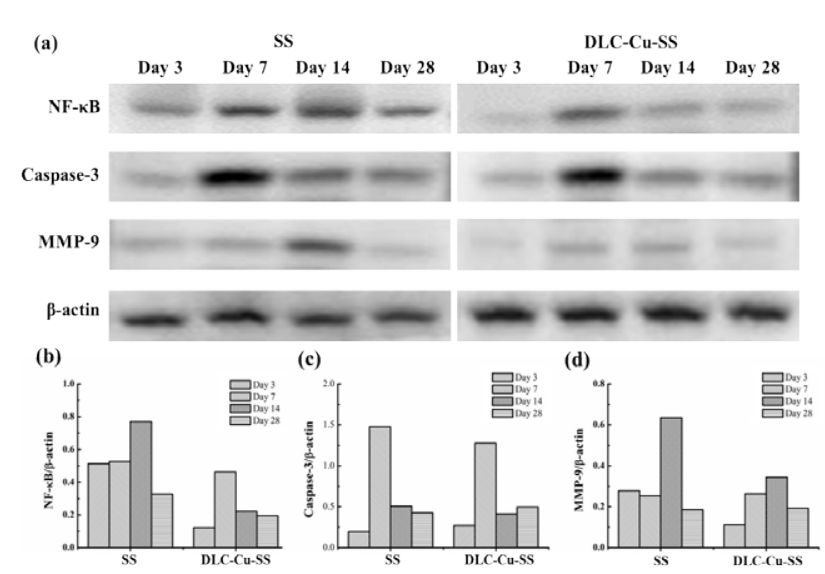

Figure 9: (a) Western blot analysis shows high expression of NF-KB at days 7 and 14, high expression of caspase- 3 at day 7 , and high expression of MMP-9 at day 14 in the SS electrode group. DLC-Cu-SS electrode group had high expressions of NF-KB and caspase-3 at day 7. (b) Densiometric analysis revealed the expression of NF-KB protein in SS electrode group was higher than that in DLC-Cu-SS electrode group at days 3 and 14. (c) Caspase-3 was highly expressed at day 7 for both groups. (d) The expression of MMP-9 in SS electrode group was higher than that in DLC-Cu-SS electrode group at day 14 . 


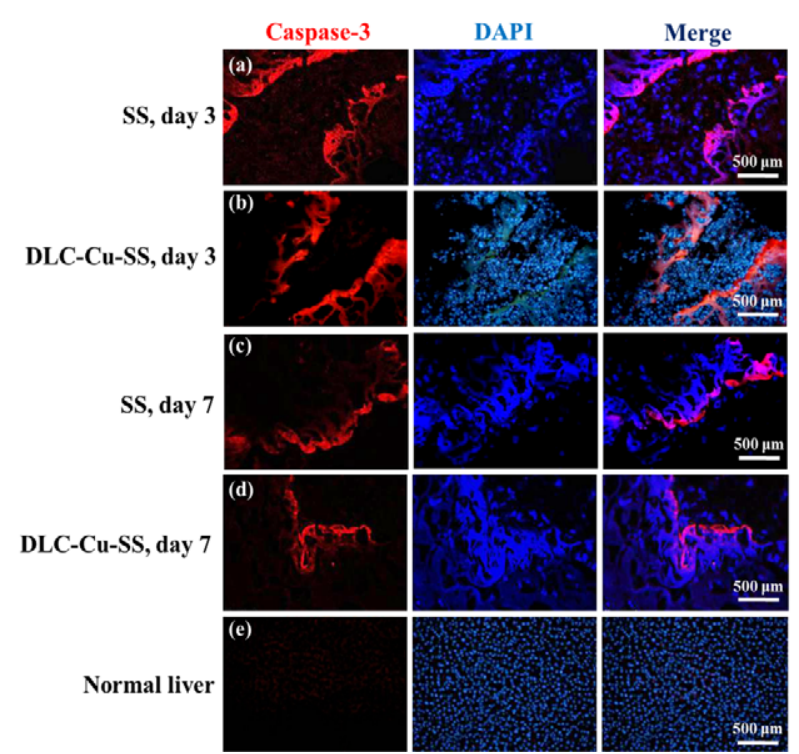

Figure 10: Most caspase-3 positive cells were found surrounding the injury site in (a) the SS and (b) DLC-Cu-SS electrode group at day 3. Caspase-3 positive cells were found also surrounding the injury site in (c) the SS and (d) DLC-Cu-SS electrode group at day 7. (e) A few cells were also positive for caspase-3 staining in normal rat liver.

electrode also caused a decrease in tissue adhesion compared with the unmodified electrode when used to make incisions on liver lobes. Mikami et al. described the properties of Au-PTFE film and Aucoating surface [23]. They found the water contact angle of Au-PTFE was larger than that for Au-coating, and the hydrophobic Au-PTFE film was less prone to tissue sticking. The same group analyzed the coagulation performances of gold, titanium, and SS bipolar forceps by comparing the amount of protein in the adhered coagulum on the tips. The surfaces of Au-plated tips were smoother than those of SS tips, and $\mathrm{Au}$ tips also caused less tissue adhesion [24]. Therefore, the DLC-CuSS electrode has a smooth and hydrophobic surface shall benefit to the anti-adhesion demand.

During the electrosurgical procedure, the surgical smoke was found when using a SS-electrode. Tissue thermographs showed that temperatures recorded for DLC-Cu-SS electrodes were significantly lower than those produced by SS-electrodes under the same RF power setting. This can be attributed to the higher electric/thermal conductivity of cupper compared to SS [25]. The modified electrode can deliver electrical energy/heat to the targeting tissue more efficiently. This property can deliver electrical energy to the target tissue more efficiently. This can prevent the overheating of the electrode and the incidence of thermal injury. However, rats received sham operation (laparotomy only), and electrosurgery with SS-electrode or DLC-CuSS electrode had no difference in postoperative pain score.

A typical injury healing process is achieved through hemostasis, inflammation, proliferation, and remodeling [26]. The SS-electrode caused a larger total injury area as well as a larger thermal area than that of the DLC-Cu-SS electrode at day 3. The size of adhesion and bleeding area was coincided with the adhesive tissues on electrodes, that is, un-modified SS-electrodes generated larger amount of sticking tissues also caused a larger adhesion and bleeding area. Histologic examinations showed a few blood cells infiltration in the adhesion and bleeding area which represented the late stage of hemostasis.
The hemostasis then initiated the early stage of inflammation, and the inflammatory phase may recruit neutrophils, macrophages, and lymphocytes to clear apoptotic cells [27]. At day 7, the outer areas of thermal injury were replaced by fibrotic tissues and the areas of adhesion and bleeding became apoptosis. The size of fibrotic area was in directly proportional to the thermal injury area. The western blot revealed that both caspase- 3 and NF- $\mathrm{kB}$ were highly expressed at day 7. The caspase cascade has intrinsic and extrinsic pathways to initiate apoptosis that ultimately induce protein and DNA degradation in cells [28]. Caspase-3, a downstream protease in the caspase cascade, is generally considered a marker of apoptosis [29]. NF- $\kappa B$ also plays an important role in apoptosis; the active NF- $\mathrm{kB}$ enters the nucleus and activates the expression of specific genes that induce the apoptosis cascade [30]. Immunofluorescence staining for caspase-3 provided further direct evidences for cell apoptosis. The results of western blot in combined with the findings of histologic inspections showed that partial direct injury areas became apoptosis at day 7 in both groups. Relative to DLC-Cu-electrodes, SS-electrodes caused more severe injury and accelerated the pace of apoptosis.

It is noteworthy that rats treated with SS-electrodes still had a large apoptotic area at day 14 . On the contrary, rats treated with DLC-Cu-SS electrodes had a relative small apoptotic area but large neogenesis area. This evidence revealed that rats in DLC-Cu-SS electrode group had a fast injury healing progress. Simultaneously, western blot analysis showed rats in the SS-electrode group secreted higher levels of NF- $\kappa \mathrm{B}$ compared with rats in the DLC-Cu-electrode group. In fact, the NF$\kappa \mathrm{B}$ is reported as a positive correlation with hepatic injury, fibrosis, and even hepatocellular carcinoma [31]. Following hepatic damage, quiescent hepatic stellate cells will be activated and trans-differentiate into extracellular matrix (ECM)-secreting myofibroblasts. Stellate cells, the major cell type involved in liver fibrosis, secrete ECM proteins, including laminins, collagens, and proteoglycans [32]. Generally, when minor hepatic injury occurs, the regenerative process may take place rapidly with the formation of fibrotic tissues [33]. Hepatic fibrosis represents the liver's wound-healing response to injury. In the later stage of liver remodeling process, stellate cells express a combination of matrix metalloproteinases (MMPs) that have the ability to degrade liver matrix. The fibrotic tissues with ECM will be degraded and finally replaced by neogenetic hepatocytes [34,35]. Rats in SS-electrode group produced high level of MMP-9. Therefore, the smaller area of fibrotic tissue associated with the use of DLC-Cu-SS electrodes indicates a better condition for liver remodeling.

At day 28, in spite of some fibrotic tissues still observed in the DLC-Cu-SS electrode group, most of the apoptotic area was replaced by hepatocyte foci which represented the late stage of liver remodeling $[36,37]$. Although the remodeling processes were similar, the total injury area of DLC-Cu-SS electrode group was smaller than the SSblade group. Moreover, the survival and activation of stellate cells and hepatic myofibroblasts are also regulated by NF- $\kappa \mathrm{B}$. Western blots showed rats treated with DLC-Cu-SS blades expressed lower NF- $\kappa B$, revealing a decrease in the number of active stellate cells and showing of the liver remodeling process as the tissue integrity was restored [33]. Overall, the animal study showed that DLC-Cu-SS electrodes caused a smaller injury area with a smaller thermal injury site than SS-electrode. The smaller thermal-injury site resulted in a decreasing fibrosis tissue and a faster neogenesis process, which was of benefit to the liverremodeling process.

\section{Conclusion}

The plating of DLC-Cu film can improve the electrode performance 
Citation: Ou KL, Cheng HY (2015) The Application of Advanced Nanostructured Film in Electrosurgical Device: Anti-Sticking Behavior and Thermal Injury. J Nanomed Nanotechnol 6: 291. doi:10.4172/2157-7439.1000291

of electrosurgical unit in terms of tissue sticking and thermal injury. The DLC-Cu-SS electrode had a less amount of sticking tissue with a lower surgical temperature during electrosurgery. The total injury area of rats treated with DLC-Cu-SS electrode was significantly smaller than those of treated with SS blades at each time points. Moreover, the DLC$\mathrm{Cu}-\mathrm{SS}$ electrode caused a relative smaller area of lateral thermal injury, a smaller area of fibrotic tissue, and a faster hepatic regeneration in rat liver. This study reveals that the plating of DLC-Cu film on electrode is an effective approach to improve the performance of electrosurgical unit.

\section{Acknowledgment}

This research was supported by the Center of Excellence for Department of Health, Executive Yuan, Taiwan (contract number MOHW103-TDU-N-211-133001) Health and Welfare Surcharge of Tobacco Products (contract number MOHW103TD-B-111-01), and Taipei Medical University Hospital (contract number TMU100AE1-B21)

\section{References}

1. Hamad GG, Curet M (2010) Minimally invasive surgery. Am J Surg 199: 263265

2. Robinson TN, Stiegmann GV (2004) Minimally invasive surgery. Endoscopy 36: 48-51.

3. Fuchs KH (2002) Minimally invasive surgery. Endoscopy 34: 154-159.

4. Kenyon TA, Urbach DR, Speer JB, Waterman-Hukari B, Foraker GF, et al (2001) Dedicated minimally invasive surgery suites increase operating room efficiency. Surg Endosc 15: 1140-1143.

5. Ikeda K, Sumiyama K, Tajiri H, Yasuda K, Kitano S (2011) Evaluation of a new multitasking platform for endoscopic full-thickness resection. Gastrointest Endosc 73: 117-122.

6. Boulay BR, Carr-Locke DL (2010) Current affairs: electrosurgery in the endoscopy suite. Gastrointest Endosc 72: 1044-1046.

7. Brill Al (2008) Bipolar electrosurgery: convention and innovation. Clin Obstet Gynecol 51: 153-158.

8. Carlander J, Johansson K, Lindström S, Velin AK, Jiang CH, et al. (2005) Comparison of experimental nerve injury caused by ultrasonically activated scalpel and electrosurgery. Br J Surg 92: 772-777.

9. Munro MG (2006) Mechanisms of thermal injury to the lower genital tract with radiofrequency resectoscopic surgery. J Minim Invasive Gynecol 13: 36-42.

10. Mattes D, Silajdzic E, Mayer M, Horn M, Scheidbach D, et al. (2010) Surgical smoke management for minimally invasive (micro)endoscopy: an experimental study. Surg Endosc 24: 2492-2501.

11. DesCoteaux JG, Picard P, Poulin EC, Baril M (1996) Preliminary study of electrocautery smoke particles produced in vitro and during laparoscopic procedures. Surg Endosc 10: 152-158.

12. Krones CJ, Conze J, Hoelzl F, Stumpf M, Klinge U, et al., (2007) Chemica composition of surgical smoke produced by electrocautery, harmonic scalpe and argon beaming - a short study. European Surgery 39: 118-121.

13. Wang K, Advincula AP (2007) "Current thoughts" in electrosurgery. Int J Gynaecol Obstet 97: 245-250.

14. Roy RK, Choi HW, Yi JW, Moon MW, Lee KR, et al. (2009) Hemocompatibility of surface-modified, silicon-incorporated, diamond-like carbon films. Acta Biomater 5: 249-256.

15. Cui FZ, Li DJ (2000) A review of investigations on biocompatibility of diamondlike carbon and carbon nitride films. Surface and Coatings Technology 131 481-487.

16. Roy RK, Lee KR (2007) Biomedical applications of diamond-like carbon coatings: a review. J Biomed Mater Res B Appl Biomater 83: 72-84.

17. Singh A, Ehteshami G, Massia S, He J, Storer RG, et al. (2003) Glial cell and fibroblast cytotoxicity study on plasma-deposited diamond-like carbon coatings. Biomaterials 24: 5083-5089.

18. Zhang L, Lv P, Huang ZY, Lin SP, Chen DH, et al. (2008) Blood compatibility of La2O3 doped diamond-like carbon films. Diamond and Related Materials 17: 1922-1926

19. Lee FP, Wang DJ, Chen LK, Kung CM, Wu YC, et al. (2013) Antibacteria nanostructured composite films for biomedical applications: microstructura characteristics, biocompatibility, and antibacterial mechanisms. Biofouling 29 : 295-305.

20. Chan YH, Huang CF, Ou KL, Peng PW (2011) Mechanical properties and antibacterial activity of copper doped diamond-like carbon films. Surface and Coatings Technology 206: 1037-1040.

21. Waterhouse A, Yin Y, Wise SG, Bax DV, McKenzie DR, et al. (2010) The immobilization of recombinant human tropoelastin on metals using a plasma-activated coating to improve the biocompatibility of coronary stents. Biomaterials 31: 8332-8340.

22. Elliott-Lewis EW, Mason AM, Barrow DL (2009) Evaluation of a new bipolar coagulation forceps in a thermal damage assessment. Neurosurgery 65: 11821187.

23. Mikami T, Minamida Y, Koyanagi I, Houkin K (2007) Novel bipolar forceps with protein repellence using gold-polytetrafluoroethylene composite film. Neurosurgery 60: ONS157-160

24. Mikami T, Takahashi A, Hashi K, Gasa S, Houkin K (2004) Performance of bipolar forceps during coagulation and its dependence on the tip material: a quantitative experimental assay. Technical note. J Neurosurg 100: 133-138.

25. Kazi SN, Duffy GG, Chen XD (2010) Mineral scale formation and mitigation on metals and a polymeric heat exchanger surface. Applied Thermal Engineering 30: 2236-2242.

26. Guo S, Dipietro LA (2010) Factors affecting wound healing. J Dent Res 89: 219-229.

27. Campos AC, Groth AK, Branco AB (2008) Assessment and nutritional aspects of wound healing. Curr Opin Clin Nutr Metab Care 11: 281-288.

28. Elmore S (2007) Apoptosis: a review of programmed cell death. Toxicol Patho 35: 495-516.

29. Pai VP, Horseman ND (2011) Multiple cellular responses to serotonin contribute to epithelial homeostasis. PLoS One 6: e17028.

30. Schmid JA, Birbach A (2008) IkappaB kinase beta (IKKbeta/IKK2/IKBKB) -a key molecule in signaling to the transcription factor NF-kappaB. Cytokine Growth Factor Rev 19: 157-165.

31. Luedde T, Schwabe RF (2011) NF-KB in the liver--linking injury, fibrosis and hepatocellular carcinoma. Nat Rev Gastroenterol Hepatol 8: 108-118.

32. Zheng ZY, Weng SY, Yu Y (2009) Signal molecule-mediated hepatic cell communication during liver regeneration. World J Gastroenterol 15: 5776-5783.

33. Friedman SL (2000) Molecular regulation of hepatic fibrosis, an integrated cellular response to tissue injury. J Biol Chem 275: 2247-2250.

34. Knittel T, Mehde M, Grundmann A, Saile B, Scharf JG, et al. (2000) Expression of matrix metalloproteinases and their inhibitors during hepatic tissue repair in the rat. Histochem Cell Biol 113: 443-453.

35. Moreira RK (2007) Hepatic stellate cells and liver fibrosis. Arch Pathol Lab Med 131: $1728-1734$

36. Alwayn IP, Verbesey JE, Kim S, Roy R, Arsenault DA, et al. (2008) A critica role for matrix metalloproteinases in liver regeneration. J Surg Res 145: 192-198.

37. Moriya K, Bae E, Honda K, Sakai K, Sakaguchi T, et al. (2011) A fibronectinindependent mechanism of collagen fibrillogenesis in adult liver remodeling Gastroenterology 140: 1653-1663. 